\title{
Effect of Divergence Time and Recombination Rate on Molecular Evolution of Drosophila INE-1 Transposable Elements and Other Candidates for Neutrally Evolving Sites
}

\author{
Jun Wang · Peter D. Keightley · Daniel L. Halligan
}

Received: 13 April 2007/ Accepted: 9 July 2007/Published online: 25 September 2007

(C) Springer Science+Business Media, LLC 2007

\begin{abstract}
Interspecies divergence of orthologous transposable element remnants is often assumed to be simply due to genetic drift of neutral mutations that occurred after the divergence of the species. However, divergence may also be affected by other factors, such as variation in the mutation rate, ancestral polymorphisms, or selection. Here we attempt to determine the impact of these forces on divergence of three classes of sites that are often assumed to be selectively unconstrained (INE-1 TE remnants, sites within short introns, and fourfold degenerate sites) in two different pairwise comparisons of Drosophila (D. melanogaster vs. D. simulans and D. simulans vs. D. sechellia). We find that divergence of these three classes of sites is strongly influenced by the recombination environment in which they are located, and this is especially true for the closer $D$. simulans vs. D. sechellia comparison. We suggest that this is mainly a result of the contribution of ancestral polymorphisms in different recombination regions. We also find that intergenic INE-1 elements are significantly more diverged than intronic INE-1 in both pairwise comparisons, implying the presence of either negative selection or lower mutation rates in introns. Furthermore, we show that substitution rates in INE-1 elements are not associated with the length of the noncoding sequence in which they are located, suggesting that reduced divergence in long noncoding sequences is not due to reduced mutation rates
\end{abstract}

Electronic supplementary material The online version of this article (doi:10.1007/s00239-007-9028-6) contains supplementary material, which is available to authorized users.

J. Wang · P. D. Keightley · D. L. Halligan ( $\square)$ Institute of Evolutionary Biology, School of Biological Sciences, University of Edinburgh, West Mains Road,

Edinburgh EH9 3JT, UK

e-mail: Daniel.Halligan@ed.ac.uk in these regions. Finally, we show that GC content for each site within INE-1 sequences has evolved toward an equilibrium value $(\sim 33 \%)$ since insertion.

Keywords INE-1 $\cdot$ Drosophila $\cdot$ Neutral evolution · Substitution rate $\cdot$ Crossing-over

\section{Introduction}

Transposable elements (TEs) are mobile, repetitive DNA sequences that are major components of many host genomes. TEs can be divided into two major classes, Class I (RNA mediated) and Class II (DNA mediated) (Berg and Howe 1989; McDonald 1993). Class I elements, or retrotransposons, are transcribed into RNA and then reverse transcribed and reintegrated into the genome, thereby duplicating the elements. Class II elements, or DNA transposons, are generally excised from one genomic site and integrated at another by a "cut-and-paste" mechanism involving a transposase (see review by Kazazian 2004). TEs comprise a large part of many eukaryotic genomes including mammals, such as human $(\sim 50 \%)$ and mouse ( $\sim 40 \%$ ) (Venter et al. 2001; Deininger and Batzer 2002; Waterston et al. 2002). In contrast, it is estimated that only $5.3 \%$ of the Drosophila melanogaster euchromatic genome is comprised of TE insertions (Quesneville et al. 2005).

The most abundant family of TEs in the D. melanogaster subgroup is INE-1 (Kapitonov and Jurka 1999, 2003). Kapitonov and Jurka (2003) speculated that INE-1 is a remnant of the $D$. virilis penelope retrotransposon, but on closer inspection it appears that INE-1 is more likely to be a family of nonautonomous DNA transposons (Pyatkov et al. 2002; Slawson et al. 2006; Yang et al. 2006). Previous analyses of this element have indicated that a burst of 
INE-1 transposition occurred in the ancestor of the $D$. melanogaster species complex $\sim 5-10 \mathrm{Myr}$ ago, and the element is thought to have remained inactive ever since, at least in the D. melanogaster lineage (Kapitonov and Jurka 1999, 2003; Singh and Petrov 2004; Singh et al. 2005a). Thus, it is reasonable to assume that many of these transposable element remnants are selectively unconstrained, and their evolution has therefore been used to assess substitution rate heterogeneity (i.e., background substitutional patterns) in the D. melanogaster genome (Singh et al. 2005a).

In the Drosophila genome, there are several other classes of nucleotide sites that have been hypothesized to be evolving close to neutrally. One class is composed of sites in short introns ( $<65$ bp in total length), outside splice control regions (base pairs 8-30 from the $5^{\prime}$ end), which have been termed the fastest-evolving intronic (FEI) sites (Halligan and Keightley 2006). A second class contains fourfold degenerate sites within coding sequences. However, there is evidence for weak selection for translational efficiency on at least some fourfold degenerate sites, resulting in codon-usage bias in Drosophila (Akashi 1995; McVean and Vieira 2001). Although sites within INE-1 elements and the two other classes of sites have all been assumed to be evolving close to neutrally in the past, there has been no attempt to compare their patterns of evolution.

Molecular evolution is expected to differ between these classes of sites for several reasons, and even if sites are completely selectively unconstrained, there are several forces that could affect their interspecies divergence. First, divergence between a pair of species is affected not only by the time since speciation but also by the time to coalescence (which is subject to stochastic variance) of those differences that are the result of polymorphisms in the common ancestor. Since the frequency of recombination affects the effective population size $\left(N_{e}\right)$ of a region of the genome via Hill-Robertson interference (Hill and Robertson 1966; Felsenstein 1974) or via the action of selective sweeps (Maynard-Smith and Haigh 1974; Kaplan et al. 1989), regions of low recombination are expected to have lower $N_{e}$ values compared to regions of high recombination. There is evidence to suggest that this is indeed the case in Drosophila (e.g., Betancourt and Presgraves 2002; Presgraves 2005; Haddrill et al. 2007). This will lead to an accelerated rate of genetic drift in regions of low recombination, and thus the coalescent time for polymorphic sites in the common ancestor will tend to be shorter, leading to lower divergence. This effect is expected to be strongest when the ratio of polymorphism (in the ancestor) to fixed differences (between species) is high. Second, interspecies divergence of unconstrained sequences can be affected by variation in the mutation rate. For example, it has been suggested that recombination itself may be mutagenic
(Lercher and Hurst 2003; Hellmann et al. 2003; Yi et al. 2004). Finally, the mutation rate may be affected by transcription-coupled repair. In this case, the mutation rate and therefore divergence would be expected to be lower in sections of the genome that are transcribed.

If sites are under weak selection, then interspecies divergence can also be affected by variation in the recombination rate, since selection acting on these sites is expected to be less effective in regions of the genome with lower effective population size (Hill and Robertson 1966; Kliman and Hey 1993; Hey and Kliman 2002). This will lead to increased divergence in low recombination rate regions if there is weak negative selection, but could produce the opposite pattern if there is a substantial fraction of positively selected substitutions. Although it is often assumed that TE remnants are unconstrained, there is mounting evidence that selection acts on at least some TEs. For example, several recent studies have indicated that some TEs may cause changes in gene regulation in plants (White et al. 1994) and mammals (McDonald 1993; Deininger et al. 2003; Jordan et al. 2003; van de Lagemaat et al. 2003). In Drosophila, two classes of non-LTR retrotransposons of Drosophila melanogaster, Het-A and TART, integrate at specific ribosomal RNA gene locations and maintain the telomeres of D. melanogaster chromosomes (Jakubczak et al. 1990; Pardue and DeBaryshe 2003). Also, the expression of the 17.6 retrotransposon in Drosophila has been shown to be crucial for the development of some tissues, including the eyes (Mozer and Benzer 1994). However, compared to mammals, there are relatively fewer fixed TE insertions in Drosophila, and segregating TE insertions appears to be at very low frequencies (Charlesworth and Langley 1989), suggesting that most TE insertions are deleterious. Thus, in contrast to mammals, it is possible that a relatively high proportion of fixed Drosophila TE insertions may be adaptive and it is interesting to test whether or not TE remnants, particularly those that persist for long periods of evolutionary time, are functional.

Furthermore, examining the molecular evolution of INE-1 elements could also shed light on the forces operating on unique noncoding DNA. For example, Haddrill et al. (2005) and Halligan and Keightley (2006) have claimed that there is a substantial amount of negative selection operating on noncoding DNA in Drosophila, based on reduced divergence in long intronic and intergenic sequences compared to synonymous sites and short introns. This raises the question of whether this pattern can instead be explained by processes other than selection, e.g., lower mutation rates in long noncoding sequences. If so, a similar reduction in divergence would be expected to be observed in INE-1 elements located within long noncoding sequences. 
In this paper, we investigate the molecular evolution of INE-1 and compare it to that of FEI and fourfold degenerate sites, by calculating the mean divergence between $D$. melanogaster and D. simulans as well as between $D$. simulans and D. sechellia for sections of the genome that have different frequencies of crossing-over. We look for evidence of variation in the substitution rate by testing for overdispersion of substitutions in INE-1 elements within each recombination category. We investigate differences between intergenic and intronic INE-1 elements. We also investigate the relationship between divergence of INE-1 elements and the lengths of the noncoding sequences in which they reside, to test whether the difference in rates of substitution observed between noncoding sequences of different lengths in Drosophila can also be observed within the INE-1 elements located in these sequences. Finally, we investigate patterns of base composition and point substitution within extant copies of INE-1 in D. melanogaster and $D$. simulans, by comparing them to inferred ancestral sequence and calculate the equilibrium GC content in the Drosophila genome.

\section{Materials and Methods}

\section{Compilation and Alignment of Sequences}

We generated three data sets of pairwise INE-1 alignments. The first (data set 1) was derived from D. melanogaster and D. simulans noncoding sequence alignments of Halligan and Keightley (2006). These noncoding alignments cover $\sim 80 \mathrm{Mb}$ of genome sequence and were initially aligned using MAVID (Bray and Pachter 2004), then refined by realigning with MCALIGN2 (Wang et al. 2006). The second data set (data set 2) was also comprised of $D$. melanogaster and D. simulans pairwise alignments, but was extracted from independently collected three-way alignments of D. melanogaster, D. simulans and D. sechellia. The final set (data set 3) was comprised of $D$. simulans and $D$. sechellia pairwise alignments obtained from the same three-way alignments used for data set 2 . The divergence between $D$. simulans and D. sechellia is much lower than that between $D$. melanogaster and $D$. simulans, allowing us to make inferences about processes whose magnitude of effect depends on divergence time. Data set 2 is a subset of data set 1 , which provides a control for data set 3 , since they are obtained from the same threeway alignments and are comprised of the same orthologous INE-1 elements.

We used a similar method to that described by Halligan and Keightley (2006) to obtain the three-way noncoding alignments used to derive data sets 2 and 3 . We obtained a list of all currently annotated $D$. melanogaster genes from
NCBI's Entrez Gene (Release 4.1; excluding RNA genes and poorly annotated genes). We then used reciprocal besthits BLAST to identify the locations of orthologous exons in the D. simulans (the April 2005 consensus assembly from the Genome Sequencing Center WUSTL School of Medicine) and D. sechellia (the October 2005 assembly from by the Broad Institute of MIT and Harvard) genome sequences. We attempted to remove any genes from the data set for which the exon/intron structure may have changed. In order to do this we chose to use only genes where we could recover all exons by the reciprocal besthits BLAST method, located in the same order as those in D. melanogaster. This should ensure that the exon/intron order in our selected genes is the same between species. Furthermore, we removed genes from the data set if the coding sequences (CDSs) were invalid (a CDS was considered valid if it started/ended with a start/stop codon, had no internals stop codons, and was a multiple of $3 \mathrm{bp}$ in length). The start and end positions of the located exons were then used to extract the adjacent intronic and intergenic sequences. Noncoding DNA sequences were only extracted if a reciprocal-best hit for the two flanking exons was found in both $D$. simulans and $D$. sechellia. As a result of this stringency, these noncoding sequences cover only $\sim 40 \mathrm{Mb}$ of the $D$. melanogaster genome sequence. Finally, we aligned orthologous sections of noncoding DNA using MAVID (Bray and Pachter 2004). We removed introns from the data set if they did not start or end with a 2-bp consensus sequence and/or if the intron sequences in D. simulans/D. sechellia started or ended with gaps (these represent incorrectly aligned sequences under the assumption that the 2-bp consensus should be aligned).

For each of the genomic noncoding sequence alignments (pairwise and three-way), we extracted the alignments of FEI sites (base pairs 8-30 from the $5^{\prime}$ end of introns $<65$ bp in length [see Halligan and Keightley 2006]). We also aligned the identified orthologous exons between $D$. melanogaster and D. simulans and orthologous exons in all three Drosophila species using the amino acid alignment obtained from CLUSTALW (Thompson et al. 1994). We then extracted the alignments of fourfold degenerate sites.

Identification and Extraction of Orthologous INE-1 Elements

We extracted orthologous INE-1 elements from the noncoding pairwise and three-way noncoding alignments as follows. (1) We removed gaps from the alignments and used the reported consensus sequence for INE-1 (Kapitonov and Jurka 2003) in RepeatMasker (http://www. repeatmasker.org) to identify INE-1 elements in all species. (2) We found the locations of the INE-1 elements 
identified with RepeatMasker within the noncoding alignments and extracted only the sections of the alignments identified as INE-1 in all species. (3) We excluded all alignments with fewer than 50 valid bases (i.e., A, T, G, or C) in any species or 100 alignment columns. (4) We attempted to exclude nonhomologous sections within the INE-1 alignments by masking sections in which there were short lengths of bases surrounded by long gaps or in which divergence was $>0.30$ (between $D$. melanogaster and $D$. simulans) or $>0.12$ (approximately three times the mean interspecies divergence between $D$. simulans and $D$. sechellia) within a 50-bp sliding window. We also excluded alignments altogether if these masked sections comprised more than $60 \%$ of the alignment.

For data set 1, we identified 1657 and 1581 INE-1 elements in step 1 from D. melanogaster and D. simulans, respectively, of which 1103 remained after step 2 . This was reduced to 613 and 353 after steps 3 and 4, respectively (312 from intergenic regions and 41 from intronic regions). For data sets 2 and 3, step 1 identified 400, 395, and 381 INE-1 elements from D. melanogaster, D. simulans, and $D$. sechellia, respectively. This was reduced to 161 after step 2. After steps 3 and 4, data set 2 comprised 149 D. melanogaster and $D$. simulans INE-1 alignments (91 intergenic and 58 intronic) and data set 3 comprised $161 \mathrm{D}$. simulans and $D$. sechellia alignments (99 intergenic and 62 intronic). See Supplementary Table 1 for details of the results from this extraction procedure.

\section{Recombination Regions}

Each data set of INE-1 elements, FEI, and fourfold degenerate sites was sorted according to cytological map location and divided into categories with high, intermediate, and low frequencies of crossing-over, and a group with no crossing-over, based on the regions described by Charlesworth (1996) and listed by Haddrill et al. (2007). These cytological locations are based on the band coding system of Charlesworth and Lapid (1989) and Charlesworth et al. (1992), which assigns approximate physical positions to loci for which information on DNA variability is available. We removed some bands in telomeric and centromeric polytene regions due to a lack of experimental recombination data. We attempted to apply estimates of recombination rate for D. melanogaster to its sister species, D. simulans and $D$. sechellia, with some modification. We have attempted to limit the effects of changes in the recombination environment by excluding sequences from a number of cytological bands due to uncertainty over changes in the recombinational environment between $D$. melanogaster and D. yakuba (Marais et al. 2004; Haddrill et al. 2007). Furthermore, we can be fairly confident that chromosome 4 has remained nonrecombining since the split of the D. melanogaster species complex (Jensen et al. 2002).

To test the association between crossing-over frequency and divergence, we assigned values $4,3,2$, and 1 to the crossing-over frequency classes, high, intermediate, low, and none, respectively, and calculated the Spearman rank correlation between divergence and crossing-over frequency value.

Estimating Divergence and Equilibrium GC Content of INE-1 Elements

All divergence estimates were corrected for multiple hits (Kimura 1980). Mean divergence for pairwise comparisons between species was calculated on a per-site basis, and 95\% confidence intervals were calculated by bootstrapping by TE element. To compare mean divergence and GC content between classes of sites, we generated 1000 bootstrap estimates of the statistic for each of the classes to be compared and then calculated the difference between each of the 1000 bootstrap estimates from the two classes and tested whether the distribution of these differences was significantly different from zero.

We inferred the polarity of substitutions along the lineages leading to the extant INE-1 sequences using the consensus sequence as an outgroup and used this information to estimate the expected proportion of bases that are $\mathrm{G} / \mathrm{C}$ at equilibrium $\left(p_{\mathrm{GC}}\right)$. Let the substitution rates from G:C to A:T pairs and A:T to G:C pairs be $r_{\mathrm{GC}}$ and $r_{\mathrm{AT}}$, respectively. Under the assumption that the pattern of substitution rates is stable through time, at equilibrium, the number of $\mathrm{G}: \mathrm{C}$ bases replaced by A:T bases will be equal to the number of A:T bases replaced by G:C pairs, so $r_{\mathrm{GC}} p_{\mathrm{GC}}=r_{\mathrm{AT}}\left(1-p_{\mathrm{GC}}\right)$. Therefore, the $\mathrm{GC}$ content at equilibrium $p_{\mathrm{GC}}=r_{\mathrm{AT}} /\left(r_{\mathrm{GC}}+r_{\mathrm{AT}}\right)$. For each recombinational category, we randomly sampled INE- 1 elements with size equal to the number of elements in the sampled category with replacement. For each sampled element, we observed the number for each type of single nucleotide substitution. We then calculated the total rate of single nucleotide substitutions and the expected GC content at equilibrium for these sampled elements. We conducted this process 1000 times and obtained the mean and 95\% confidence intervals for rates of different nucleotide substitutions and the expected GC content at equilibrium.

Measurement of Dispersion of Substitutions Among INE-1 Elements

If substitutions result from independent mutations occurring along each lineage since time of speciation without 
any effect of selection, then each INE-1 element is expected to accumulate substitutions at the same rate. The observed number of nucleotide differences in each element is then expected to follow the same binomial distribution (i.e., the probability of observing a difference at a site will be constant across elements). We tested this null hypothesis by assuming that the probability of observing a difference at any site was equal to the observed mean divergence (uncorrected for multiple hits). For each recombination category in each data set, we generated 1000 simulated data sets consisting of divergence estimates for sequences with the same length distribution as the actual data and calculated the variance of divergence between elements for each simulation to generate a null distribution of variance of divergence among elements. If substitutions are overdispersed, then the observed variance for the given data set will be larger than that expected under the null hypothesis of equal rates.

\section{Results}

Evaluation of Mean Divergence Controlled for Recombination Rate

Previous analysis of INE-1 elements in D. melanogaster (Singh et al. 2005a) has suggested that there are differences in rates of substitutions in INE-1 copies among different recombination environments. To investigate this further, we divided INE-1, FEI, and fourfold degenerate site alignments into four crossing-over frequency categories (high, intermediate, low, and no crossing-over) and compared divergence of each site class within and among categories. We found that the divergence of fourfold degenerate sites is always lower than that of FEI sites (see Fig. 1). This confirms previous observations (Halligan and Keightley 2006) and is consistent with reports that some fourfold degenerate sites are under weak selective constraints in Drosophila (Akashi 1995; McVean and Vieira 2001).

Interestingly, Spearman rank correlations between the divergence of FEI/fourfold sites and crossing-over frequency are positive in all three data sets (Table 1). These correlations are significant for the cases of all sequence categories for data set 3 and FEI sites in data set 2. There are several possible explanations for the positive correlation between divergence and crossing-over frequency, such as more effective/stronger positive selection in high recombination regions, mutagenic effect of recombination, and differences in the level of the ancestral polymorphism (see Discussion for details).

However, divergence patterns of INE-1 elements are quite different (Table 1). In data sets 1 and 2, there is a
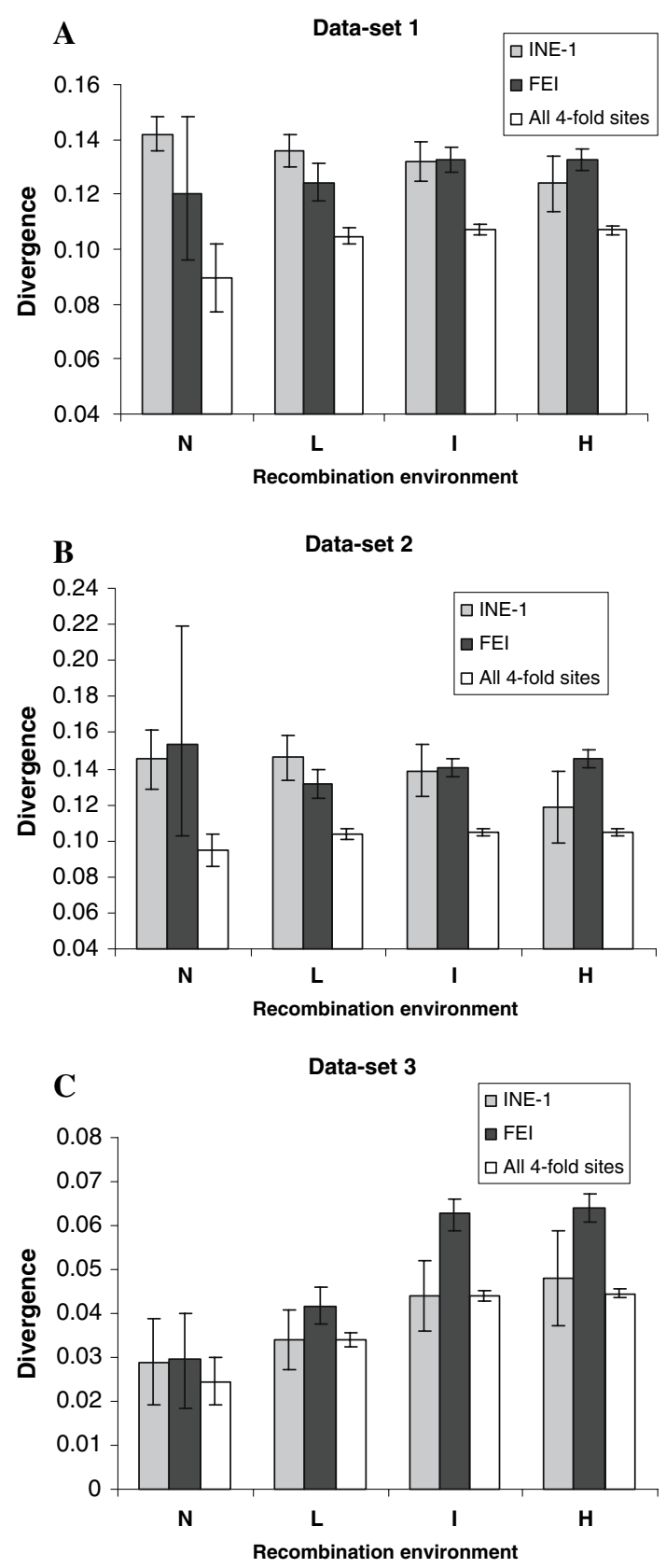

Fig. 1 Mean divergence ( $\pm 95 \%$ confidence intervals) of INE-1, FEI, and fourfold sites from (A) data set 1, (B) data set 2, and (C) data set 3. Within each data set, the three classes of nucleotide sites were subdivided into categories according to frequency of crossing-over: high $(\mathrm{H})$, intermediate $(\mathrm{I})$, low $(\mathrm{L})$, and no $(\mathrm{N})$ crossing-over. Ninetyfive percent confidence intervals were obtained by bootstrapping by element/intron/gene

negative correlation between divergence and crossing-over frequency for INE-1 elements (significantly so for the larger data set 1 ), but a significantly positive correlation for INE-1 elements in data set 3. Furthermore, in regions of high recombination, INE-1 elements have a lower mean divergence than FEI sites (in all three data sets), and this is significant for data sets $2(p=0.042)$ and $3(p=0.01)$. It is 
Table 1 Spearman correlation between divergence and rate of recombination for INE-1, FEI, and fourfold degenerate sites: 4, 3, 2, and 1 are values for high-frequency, intermediate-frequency, lowfrequency, and no crossing-over, respectively

\begin{tabular}{llll}
\hline Data set & \multicolumn{3}{l}{ Spearman correlation coefficient $(r)$} \\
\cline { 2 - 4 } & INE-1 & FEI & Fourfold \\
\hline 1 & $-0.171^{* *}$ & 0.153 & 0.015 \\
2 & -0.153 & $0.054^{* *}$ & 0.003 \\
3 & $0.196^{*}$ & $0.107^{* * *}$ & $0.12 * *$ \\
\hline
\end{tabular}

Note. ${ }^{*} p<0.05 ; * * p<0.01 ; * * * p<0.001$

possible to explain the latter result without the need to invoke selection on either FEI sites or INE-1 elements, if INE-1 elements were less polymorphic than FEI sites in the common ancestor.

In data set 3, consistent with the results for FEI and fourfold degenerate sites, there is a positive correlation between divergence and crossing-over frequency for INE1. This result could be explained by the fact these species are so closely related that the effect of increased coalescence time in the common ancestor in regions of high crossing-over frequency dominates any other effects that could potentially cause a negative correlation.

It is worth mentioning that GC content and recombination rate are negatively correlated for the $\mathrm{X}$ chromosome, while the opposite is true for the autosomes (Singh et al. 2005b). It is therefore possible that our estimates of the correlation between divergence and crossingover frequency are influenced by grouping X-linked elements and autosomal elements together. To investigate this, we divided the 353 INE- 1 elements from data set 1 (since it is the largest) into subsets of X-linked elements (46) and autosomal elements (307). We then repeated the analysis on the autosomal elements only and obtained very similar results to those for the grouped autosomal and Xlinked elements. Unfortunately, there were insufficient X chromosome data to obtain reliable estimates of the correlation between divergence and crossing-over frequency, but we did not find any significant difference in mean divergence and GC content between X-linked and autosomal elements (data not shown).

\section{Overdispersion of INE-1 Substitutions}

Variation in the substitution rate can be assessed by testing for overdispersion of substitutions. This variation could be caused by mutation rate variation, positive or negative selection that varies between elements, or the contribution of polymorphism in the ancestor to divergence between species. The latter effect is expected to be strongest when
Table 2 Test of over-dispersion of substitutions in INE-1 elements for different crossing-over categories in data sets 1,2, and 3

\begin{tabular}{|c|c|c|c|c|}
\hline Data set & Category & $N$ & $\begin{array}{l}\text { Mean null variance } \\
\text { [95\% range] }\end{array}$ & $\begin{array}{l}\text { Observed } \\
\text { variance }\end{array}$ \\
\hline \multirow[t]{4}{*}{1} & $\mathrm{H}$ & 52 & $0.0007[0.0004-0.0010]$ & $0.0010 * *$ \\
\hline & I & 100 & $0.0009[0.0004-0.0009]$ & $0.0009 *$ \\
\hline & $\mathrm{L}$ & 127 & $0.0007[0.0005-0.0009]$ & $0.0011 * * *$ \\
\hline & $\mathrm{N}$ & 66 & $0.0007[0.0004-0.0010]$ & 0.0007 \\
\hline \multirow[t]{4}{*}{2} & $\mathrm{H}$ & 27 & $0.0015[0.0006-0.0025]$ & $0.0027 *$ \\
\hline & I & 43 & $0.0015[0.0007-0.0023]$ & 0.0017 \\
\hline & $\mathrm{L}$ & 60 & $0.0014[0.0008-0.0020]$ & $0.0021 *$ \\
\hline & $\mathrm{N}$ & 16 & $0.0012[0.0001-0.0023]$ & 0.0008 \\
\hline \multirow[t]{4}{*}{3} & $\mathrm{H}$ & 28 & $0.0006[0.0002-0.0009]$ & $0.0011 * *$ \\
\hline & I & 47 & $0.0005[0.0003-0.0008]$ & $0.0019 * * *$ \\
\hline & $\mathrm{L}$ & 67 & $0.0003[0.0002-0.0005]$ & $0.0006 * * *$ \\
\hline & $\mathrm{N}$ & 16 & $0.0003[0.0001-0.0005]$ & $0.0013 * * *$ \\
\hline
\end{tabular}

Note. Within each data set, INE-1 elements were subdivided according to different recombination environments. We show the null distribution of variance in divergence uncorrected for multiple hits with a constant substitution rate and the observed variance. $* p<0.05$; $* * p<0.01 ; * * * p<0.001$

the ratio of ancestral polymorphism to divergence is highest. We tested for overdispersion in each crossing-over category of each data set by comparing the observed variance in divergence across elements to the expected, assuming a constant substitution rate and the same alignment length distribution. We found evidence for overdispersion of the substitution rate in 9 of 12 cases (Table 2), the strongest evidence for overdispersion coming from elements in data set 3 , on the basis that the ratio of observed to expected variance was highest and the $p$-values were the most significant. Although all of the abovementioned processes could contribute to the patterns observed, only the third explanation, i.e., a contribution from ancestral polymorphism, clearly predicts that overdispersion would be strongest in the closer pairwise comparison of $D$. simulans and $D$. sechellia.

\section{Comparisons Between Intergenic and Intronic INE-1 Elements}

One possible factor that could explain some overdispersion of substitutions, because of either differences in the mutation rate or differences in the level of selection, is transcription. A recent study in mammals found that transposable elements in introns have a slightly lower mean divergence than those in intergenic DNA (Gaffney and Keightley 2006). We tested for differences in substitution rate between intergenic and intronic INE-1 alignments in each data set. Consistent with the observations in 
Table 3 Mean divergence and GC content of intergenic and intronic INE-1 elements for data sets 1, 2, and 3: 95\% confidence intervals for divergence and GC content were calculated by bootstrapping 1000 times by INE-1 element

\begin{tabular}{lllll}
\hline Data set & Class & $N$ & Mean divergence [95\% CI] & GC content [95\% CI] \\
\hline 1 & Intergenic & 312 & $0.135[0.131-0.139]$ & $0.376[0.372-0.380]$ \\
& Intronic & 41 & $0.123[0.112-0.135]$ & $0.371[0.361-0.381]$ \\
2 & Intergenic & 91 & $0.153[0.142-0.163]$ & $0.378[0.368-0.388]$ \\
& Intronic & 58 & $0.123[0.114-0.132]$ & $0.385[0.372-0.398]$ \\
3 & Intergenic & 99 & $0.039[0.034-0.045]$ & $0.375[0.363-0.388]$ \\
& Intronic & 62 & $0.033[0.028-0.039]$ & $0.391[0.378-0.404]$ \\
\hline
\end{tabular}

mammals, intergenic INE-1 elements have somewhat higher mean divergence than intronic elements, and this is significant for data sets $1(p<0.05)$ and $2(p<0.01)$ but not $3(p=0.28)$ (Table 3$)$. Furthermore, the difference between intergenic and intronic elements is consistent within each recombination category for all data sets (data not shown), suggesting that this result is not due to differences in levels of crossing-over between intronic and intergenic regions. Additionally, there are no significant differences in mean GC content or mean length between intergenic and intronic elements in all three data sets.

Correlation Between INE-1 Divergence and Length of Noncoding Sequence

It has previously been shown that there is a strong negative correlation between intron length and divergence in Drosophila (Haddrill et al. 2005; Halligan and Keightley 2006), and a similar pattern has also been found in intergenic sequences (Halligan and Keightley 2006). If these length correlations are the result of lower mutation rate in long noncoding sequences, or other general factors affecting all sites within long noncoding sequences, we would predict that INE-1 elements in long noncoding sequences would have lower divergence than those in short noncoding sequences. However, we found no significant correlation between INE-1 element divergence and length of the noncoding sequence in which the elements are located in any of the data sets (Spearman correlation $r=$ $0.061, p=0.25$, for data set $1 ; r=0.072, p=0.18$, for data set $2 ; r=0.051, p=0.28$, for data set 3 ). Note that there is a difference in sequence lengths between intronic and intergenic sequences, so we separated intronic sequences from intergenic sequences, and calculated the correlation of INE-1 divergence and noncoding sequence length separately. However, we did not found any relationship between these two factors for data sets 1 (intergenic, Spearman correlation $r=-0.001, p=0.98$; intronic, Spearman correlation $r=0.25, p=0.12$ ), 2 (intergenic, Spearman correlation $r=0.01, p=0.88$; intronic,
Spearman correlation $r=0.26, p=0.16$ ), and 3 (intergenic, Spearman correlation $r=-0.02, p=0.64$; intronic: Spearman correlation $r=0.18, p=0.34)$. It is worth mentioning that correlation is always close to zero for intergenic sequences but positive and quite strong for intronic sequences (although not significantly). This suggests that intronic and intergenic sequences may evolve in (slightly) different manners. Our results suggest that the difference in divergence between short and long noncoding sequences is not a result of any factors, such as mutation rate, that have general differential effects on long vs. short noncoding sequences and, instead, supports the conclusion that the difference is due to stronger or more extensive negative selection in long noncoding sequences.

\section{Evolution of GC Content of INE-1}

If the ancestral INE-1 sequence were available, we would be able to polarize substitutions in the D. melanogaster and D. simulans lineages. Although previous studies have assumed the reported consensus is a fair approximation to the ancestral sequence (Singh and Petrov 2004; Singh et al. 2005a), none have tested this assumption. Using data set 1 , we reconstructed the consensus sequences of INE-1 from the extant copies in D. melanogaster and D. simulans separately. The divergence between these two reconstructed consensus sequences is very low $(\sim 0.005)$. None of the four pairwise comparisons between the extant sequences of either species and the consensus sequences constructed from either species are significantly different from one another (Table 4). This implies that the consensus sequence of INE-1 is a good approximation to the ancestral sequence.

Given the ancestral sequence and the extant copies of INE-1, we were able to investigate how GC content has changed in each species. We calculated GC content in the consensus and mean GC content in extant sequences in $D$. melanogaster within 50-bp sliding windows across each INE-1 element. Figure 2 shows these results for high and no crossing-over categories for elements in D. melanogaster 
Table 4 Mean divergence between extant INE-1 copies in D. melanogaster and $D$. simulans and the consensus sequences constructed from each species

\begin{tabular}{lll}
\hline Consensus species & $\begin{array}{l}\text { Extant copy } \\
\text { species }\end{array}$ & $\begin{array}{l}\text { Mean divergence } \\
{[95 \% \text { CI] }}\end{array}$ \\
\hline D. melanogaster & D. melanogaster & $0.1879[0.1831-0.1928]$ \\
D. melanogaster & D. simulans & $0.1841[0.1807-0.1875]$ \\
D. simulans & D. melanogaster & $0.1878[0.1830-0.1927]$ \\
D. simulans & D. simulans & $0.1839[0.1804-0.1873]$ \\
\hline
\end{tabular}

from data set 1 (plots for other categories showed very similar patterns; see Supplementary Fig. 1). Although GC content appears to have reduced in the D. melanogaster and $D$. simulans lineages since their common ancestor, there is substantial variation across the INE-1 element. GC content in the consensus varies between $10 \%$ and $60 \%$, whereas mean GC content of the extant copies shows much less variation. By polarizing substitutions in the D. melanogaster and $D$. simulans lineages using parsimony with the consensus sequence as an outgroup, we calculated lineagespecific rates of substitution. We estimated rates for each of six types of nucleotide substitution (two transitions and four transversions) in the different crossing-over categories (Fig. 3) using data set 1 only (since this contains the most data). There is no significant heterogeneity in substitution rates across crossing-over categories (ANOVA: $p=0.99$ for both $D$. melanogaster and $D$. simulans), however, rates of the different types of substitutions do vary significantly. In particular, the G:C $\rightarrow$ A:T rate is $\sim 2.5$-fold higher than any other rate, and substitution rates generally are biased toward A:T. If we assume that this pattern is stable in both species through time, we can estimate the expected GC content at equilibrium for INE-1 elements. We did not find any difference in expected GC content at equilibrium among the four recombinational environments in both species (see Supplementary Table 2). Combining the data from different recombination environments, the expected GC content at equilibrium is estimated to be $32.3 \%$ (95\% CI, 29\%-35\%) for D. melanogaster and $33.7 \%$ (95\% CI, 31\%-37\%) for D. simulans.

Under the neutral model of nucleotide substitutions and a uniform mutation rate, the ratio of transitions (ts) to transversions (tv) is expected to be 1:2 (because there are twice as many possible transversions). Combining elements from the four recombinational categories, we observed 5582 transitions and 5711 transversions in the $D$. melanogaster lineage and 5653 transitions and 5812 transversions in the $D$. simulans lineage. The ts:tv ratios (1:1.02 in D. melanogaster; $1: 1.03$ in D. simulans) are clearly different from 1:2, as expected under a uniform mutation rate (binomial exact test: $p<<0.001$ ). They are also significantly different from the ts:tv ratio observed for

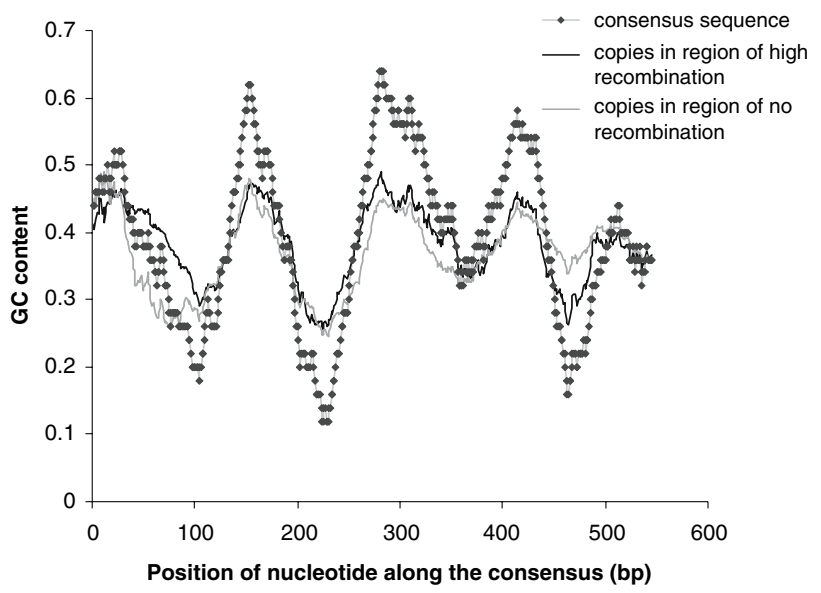

Fig. 2 Mean GC content of consensus sequence (light-gray line with dark dots) and the orthologous INE-1 extant remnants in Drosophila for each nucleotide site along the consensus sequence in 50-bp sliding windows in data set 1 for $D$. melanogaster. Within the data set, we only show results for INE-1 in regions of high (black line) and no (gray line) recombination

helena element in Drosophila, 1:1.22 ([Petrov and Hartl 1999] binomial exact test: $p<0.001)$. Transitions seem to be more favored in INE-1. This increased transition rate is mainly attributable to $\mathrm{G}: \mathrm{C} \rightarrow \mathrm{A}: \mathrm{T}$ substitutions. However, our estimates of the ts:tv ratio are very close to that estimated for synonymous substitutions between $D$. melanogaster and D. simulans in two nuclear genes, Adhr and Adh (roughly 1:1 [Moriyama and Powell 1997]). They are also close to the ts:tv ratio (about 1:1) observed in noncoding polymorphisms in Drosophila (Moriyama and Powell 1996). This suggests that transition bias may be a general pattern for relatively unconstrained sequences in Drosophila.

\section{Heterogeneity of Evolution of GC Content} Along INE-1

We have shown that GC content varies dramatically along the INE-1 consensus sequence and that regions of high ancestral GC content appear to be evolving toward reduced GC content, and vice versa. Thus, it is possible that regions with high ancestral GC content will show different substitution patterns compared to regions with low ancestral GC content. To investigate this, we divided the INE-1 consensus sequence (594 bp in length) into 12 nonoverlapping 50-bp segments and calculated the ancestral GC content within each segment. We arbitrarily split these segments into those with high GC $(>40 \%)$, moderate GC $(>30 \%,<40 \%)$, and low GC $(<30 \%)$ content (combining elements from the four recombinational environments). We then examined substitution patterns in the high and low GC content categories in D. melanogaster and D. simulans for 
A

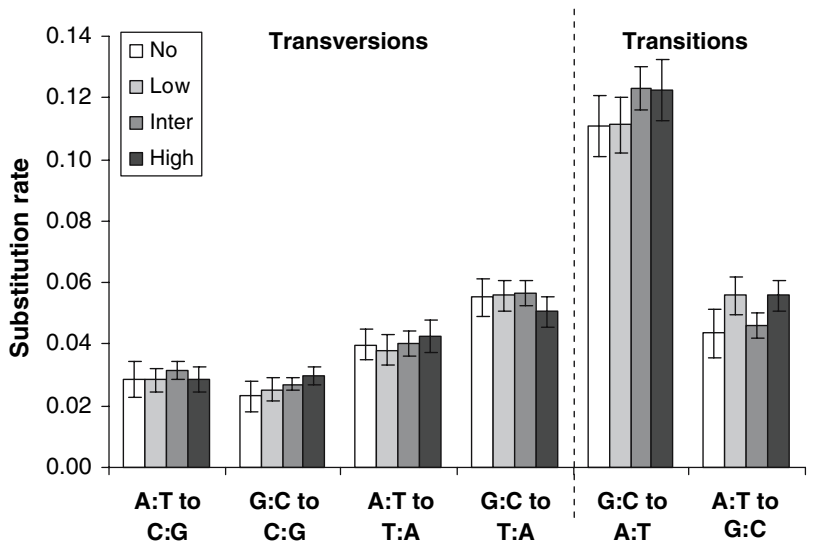

B

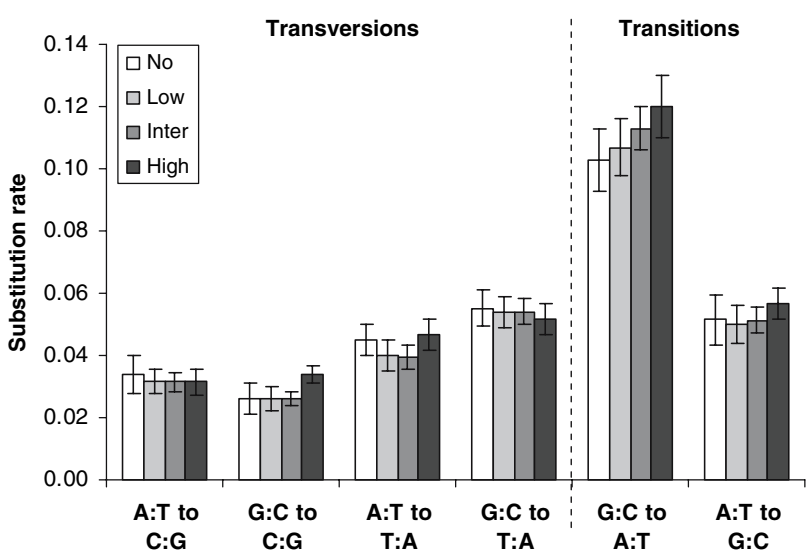

Fig. 3 Mean substitution rate $( \pm \mathrm{SE})$ of six different types of nucleotide substitutions for INE-1 elements between $D$. melanogaster and $D$. simulans from data set 1 . Substitution rates were estimated by comparing the ancestral (consensus) sequence to extant INE-1 elements in (A) D. melanogaster and (B) D. simulans. The rates are shown for the four different crossing-over frequency categories (no, low, intermediate, and high crossing-over, in order)

data set 1, using the INE-1 consensus sequence as an outgroup.

This analysis revealed no significant differences between the high and the low categories for the six different substitution rates in both species (Fig. 4). This is what would be expected if the INE-1 sequences were evolving neutrally. However, differences in GC content between these two GC content categories lead to differences in the relative numbers of GC $\rightarrow \mathrm{AT}$ and $\mathrm{AT} \rightarrow \mathrm{GC}$ substitutions. For example, in D. melanogaster, we observed $1981 \mathrm{GC} \rightarrow$ AT and 1176 AT $\rightarrow$ GC substitutions in the high ancestral GC content category (leading to a reduction in the number of GC bases), whereas in the low GC content category we observed $837 \mathrm{GC} \rightarrow$ AT and 955 AT $\rightarrow$ GC substitutions (leading to an increase in the number of GC bases).

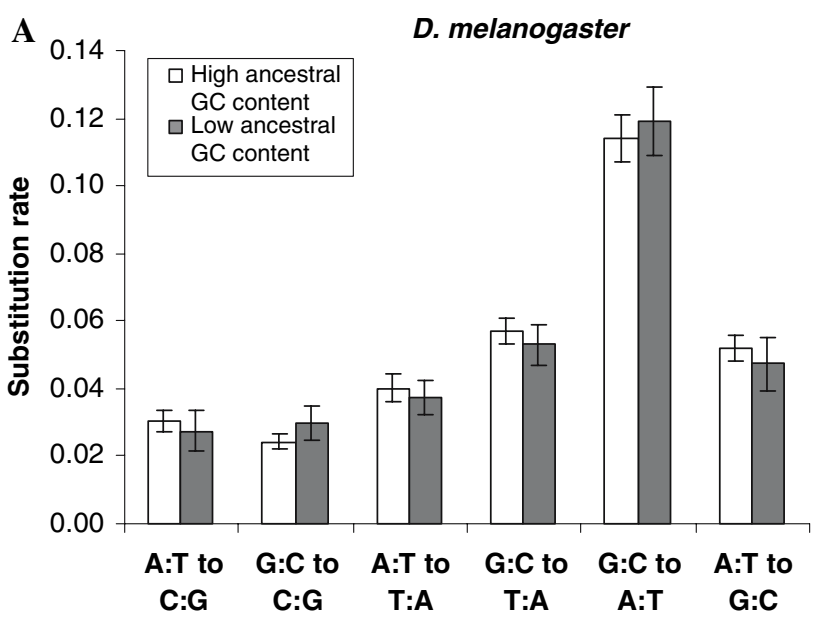

D. simulans

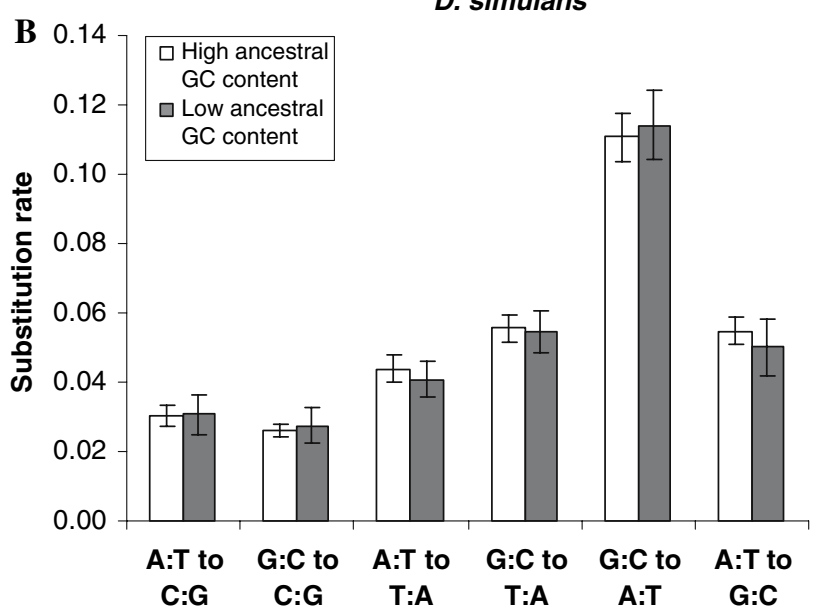

Fig. 4 Mean substitution rate $( \pm \mathrm{SE})$ of six different types of nucleotide substitutions for INE-1 in (A) D. melanogaster and (B) $D$. simulans from data set 1 . Here, INE-1 consensus sequence is divided into regions with high ancestral GC content $(>40 \%)$ and regions with low ancestral GC content $(<30 \%)$. We compare substitutional patterns between the two regions in each species

\section{Discussion}

We investigated rates and patterns of substitution in three different classes of sites of the Drosophila genome, which previous studies have hypothesized to be evolving close to neutrally. We compared evolution of remnants of the most abundant class of TEs in the D. melanogaster subgroup (INE-1) with sites within short introns (termed FEI sites) and fourfold degenerate synonymous sites. We found strong evidence for varying rates of substitution between these site classes, between different recombination environments, and between intronic and intergenic sequences. Furthermore, the patterns are inconsistent between pairs of species that differ in their divergence times. These observations could be attributable to various processes, including time to coalescence in the common ancestor of 
differences resulting from ancestral polymorphisms, mutation rate variation, and selection. These possible explanations in relation to each finding are discussed below.

We found that the mean divergence of fourfold sites is lower than that of FEI sites in all data sets and crossingover categories. This supports previous observations that at least some fourfold degenerate sites are under weak selection for protein translation efficiency (Akashi 1995; McVean and Vieira 2001) and suggests that FEI sites are evolving closer to neutrally (Halligan and Keightley 2006).

We found a positive correlation between rate of crossing-over and divergence in FEI/fourfold sites in all three data sets. A similar pattern has previously been observed for the divergence of short introns between D. melanogaster and D. yakuba (Haddrill et al. 2007). A positive correlation has also been shown in other organisms for fourfold degenerate sites (Lercher and Hurst 2002; Waterston et al. 2002; Hardison et al. 2003; Hellmann et al. 2003). In contrast, Shapiro et al. (2007) found no relationship between recombination rate and synonymous site divergence between $D$. melanogaster and $D$. simulans. The correlation observed for FEI/fourfold sites in our data also contrasts with the results of Begun and Aquadro (1992), who found no correlation between the coefficient of exchange and divergence for 20 genic regions between $D$. melanogaster and $D$. simulans. It also contrasts with results obtained for amino acid sites in Drosophila (Betancourt and Presgraves 2002; Presgraves 2005; Haddrill et al. 2007), in which divergence was highest in regions lacking recombination. It has been suggested that the latter results are due to variation in the effectiveness of selection, which is expected to be lower in regions with less frequent crossing-over due to reduced $N_{e}$. This would result in a higher rate of substitution for sites under weak negative selection in these regions. In contrast, the patterns observed for FEI/fourfold sites in our data sets could be explained by weak positive selection on these sequences in regions of no crossing-over, such that divergence is inflated above the neutral expectation in regions of high recombination. Alternatively, the patterns observed could be explained by a mutagenic effect of recombination, which has been suggested previously to explain a similar pattern observed for fourfold degenerate sites in mice (Lercher and Hurst 2002; Waterston et al. 2002; Hardison et al. 2003) and in humans (Hellmann et al. 2003). Finally, they may result from differences in the mean coalescence time among recombination rate regions. Total divergence between a pair of species reflects the time since speciation plus the coalescence time of polymorphisms present in the common ancestor. Lack of recombination leads to a lower effective population size because of Hill-Robertson interference and therefore increased rates of genetic drift. Therefore, regions with a low frequency of crossing-over would be expected to have the shortest mean coalescence time. All else being equal, this will result in lower apparent divergence for sequences in regions lacking recombination. This interpretation is supported by the fact that the magnitude of the relative differences between recombination rate regions is higher in the D. simulans vs. D. sechellia pairwise comparison, for which the ratio of polymorphism in the ancestor to divergence is highest. One should note that the ecology of the three species is very different, thus the effective population size $N_{e}$ is likely to be different between them. Indeed, $N_{e}$ tends to be relatively smaller for $D$. sechellia and larger in D. simulans. But we argue that this could affect only the magnitude of the correlation coefficients, and not the general pattern of our results.

We observed markedly different patterns of divergence for INE-1 elements among the three data sets. In regions of high crossing-over frequency, INE-1 elements have a lower mean divergence than FEI sites (in all three data sets), and this is significant for data sets $2(p=0.042)$ and $3(p=$ 0.01 ). This may be a result of weak negative selection acting on some/all INE-1 elements in this category, reducing divergence relative to the neutral expectation. The lack of difference between intermediate, low and no crossing-over frequency categories could then be attributed to less effective selection or the lack of any selection on these elements at all. However, the result could also be attributable to a process whereby the coalescence time of polymorphisms present in the common ancestor tends to be shallower for INE-1 elements than for FEI sites. If TE insertions are relatively recent, they may not have been at mutation-drift balance in the common ancestor. This would reduce the expected coalescence time and therefore apparent divergence for these elements. Thus, it is not necessary to invoke selection on INE-1 elements to explain these results. However, we also found a significantly negative correlation between divergence and crossing-over frequency in data sets 1 and 2 (not 3). This result is not easily explained by differences in coalescence times, which would predict the opposite relationship, unless INE-1 elements in the high crossing-over frequency category tend to be younger. However, INE-1 elements in high crossingover frequency categories are not more closely related to the consensus sequence (see Fig. 2), which argues against this interpretation. Therefore, there is some weak support for negative selection on INE-1 elements in highly recombining regions, at least between $D$. melanogaster and D. simulans.

We found strong evidence for overdispersion of substitutions for INE-1 remnants in 9 of 12 crossing-over categories from the three data sets. This could be explained by variation in time to coalescence of ancestral polymorphisms, which is subject to stochastic variance, but is also, 
at least, partly explained by differences between intergenic and intronic elements. In all three data sets we found that intergenic INE-1 elements evolve more rapidly than intronic ones. This pattern has also recently been observed in rodent TEs (Gaffney and Keightley 2006). Furthermore, this is apparent within crossing-over categories, suggesting that it is not an artifact of differences in rates of recombination between intergenic and intronic regions. There are several possible explanations for this result. First, sites in introns may experience a lower mutation rate, e.g., as a result of transcription-coupled repair (TCR), since sites in intronic regions are transcribed, whereas those in intergenic regions are not. This mechanism has been found in bacteria, yeast, and mammals (Deaconescu et al. 2006; LePage et al. 2000) but not in Drosophila. Second, there may be negative selection on transcribed DNA generally. For example, it has been shown that selection in introns of the alcohol dehydrogenase locus (Adh) of Drosophila pseudoobscura helps maintain secondary structure of pre-mRNA (Kirby et al. 1995). Finally, there may be greater negative selection acting on intronic INE-1 because, for example, intronic INE-1 elements are more likely to be co-opted for a function than intergenic elements.

Finally, we have investigated the pattern of base composition of INE-1 since insertion by aligning extant remnants with the consensus sequence (after establishing that this is likely to be a reasonable approximation of the true ancestral sequence). We found that substitutions since insertion have tended to be biased toward $\mathrm{A}$ and $\mathrm{T}$ nucleotides in both $D$. melanogaster and D. simulans. These patterns are consistent with previous results for INE-1 elements (Singh et al. 2005a) and helena elements (Petrov and Hartl 1999), also in Drosophila. Under the assumption that this is a result of a mutation bias, rather than purifying selection, we estimated the expected equilibrium GC content to be $33.1 \%$ (95\% CI, 30\%-36\%; combining crossingover regions and data from D. melanogaster and D. simulans). This is consistent with other estimates of the equilibrium GC content for putatively neutrally evolving sites (Petrov and Hartl 1999) and with an estimate of equilibrium GC content for low recombination regions in Drosophila (33.0\%), based on substitution rates among paralogous copies of INE-1 in D. melanogaster (Singh et al. 2005a). However, we did not find any differences in expected GC content at equilibrium among the four recombinational environments, which was shown in a previous study (Singh et al. 2005a). This is possibly caused by a lack of power due to the small sample size of INE-1 elements in regions of no crossing-over in our data.

In our data, we have observed differences in interspecies divergence among nucleotide site types, among regions with different frequencies of crossing-over. Furthermore, in some cases the relative strength and direction of these patterns vary depending on species compared. We also found evidence for overdispersion of substitutions between INE-1 elements, especially in the close $D$. simulans/D. sechellia comparison. However, one should note that applying recombination estimates in D. melanogaster to other species may bias our results. We conclude that the majority of the patterns observed can be explained by differences in time to coalescence of polymorphisms in the common ancestor. Furthermore, this process can explain the differences observed between species comparisons, since the magnitude of this effect is expected to be stronger when species are less diverged (e.g., D. simulans and $D$. sechellia). Although a mutagenic effect of recombination could produce similar patterns of evolution, here we argue that it is not necessary to invoke this, given that the process described above is likely to be operating. Additionally, a mutagenic effect of recombination could not explain the difference in the magnitude of the observed effect between the two different pairwise comparisons (D. melanogaster vs. D. simulans and D. simulans vs. D. sechellia). However, we argue that some observations, i.e., the faster evolutionary rate for intergenic INE-1 elements than intronic elements, the negative correlation between divergence and frequency of crossing-over for INE-1 between D. melanogaster and D. simulans, and the faster evolution of FEI sites than for fourfold sites, is difficult to explain by either variation in time to coalescence resulting from polymorphisms in the common ancestor or mutagenic effect of recombination. Instead, we suggest that these observations may result from variation in strength of negative or positive selection among elements.

Acknowledgments We are grateful to the Genome Sequence Center, WUSTL School of Medicine, the Broad Institute of MIT and Harvard, and the Berkeley Drosophila Genome Project for providing the genome sequences we analyzed in this study. We also thank Flybase and NCBI for providing genome annotation data. We thank Toby Johnson, Daniel Gaffney, and Brian Charlesworth for helpful comments. J.W. was supported by the Dorothy Hodgkin Postgraduate Studentship Award. Funding for D.L.H. was provided by the Wellcome Trust.

\section{References}

Akashi H (1995) Inferring weak selection from patterns of polymorphism and divergence at "silent" sites in Drosophila DNA. Genetics 139:1067-1076

Aquado CF, Begun DJ, Kindahl EC (1994) Selection, recombination, and DNA polymorphism in Drosophila. In: Golding B (ed) Nonneutral evolution: theories and molecular data. Chapman and Hall, London, pp 46-56

Begun DJ, Aquadro CF (1992) Levels of naturally occurring DNA polymorphism correlate with recombination rates in D. melanogaster. Nature 356:519-520

Berg DE, Howe MM (1989) Mobile DNA. ASM Press, Herndon, VA

Betancourt AJ, Presgraves DC (2002) Linkage limits the power of natural selection in Drosophila. Proc Natl Acad Sci USA 99:13616-13620 
Bray N, Pachter L (2004) MAVID: constrained ancestral alignment of multiple sequences. Genome Res 14:693-699

Charlesworth B (1996) Background selection and patterns of genetic diversity in Drosophila melanogaster. Genet Res 68:131-149

Charlesworth B, Langley CH (1989) The population genetics of Drosophila transposable elements. Annu Rev Genet 23:251287

Charlesworth B, Lapid A (1989) A study of ten transposable elements on $\mathrm{X}$ chromosomes from a population of Drosophila melanogaster. Genet Res 54:113-125

Charlesworth B, Lapid A, Canada D (1992) The distribution of transposable elements within and between chromosomes in a population of Drosophila melanogaster. I. Element frequencies and distribution. Genet Res 60:103-114

Deaconescu AM, Chambers AL, Smith AJ, et al. (2006) Structural basis for bacterial transcription-coupled DNA repair. Cell 124:507-520

Deininger PL, Batzer MA (2002) Mammalian retroelements. Genome Res 12:1455-1465

Deininger PL, Moran JV, Batzer MA, Kazazian HH Jr (2003) Mobile elements and mammalian genome evolution. Curr Opin Genet Dev 13:651-658

Felsenstein J (1974) The evolutionary advantage of recombination. Genetics 78:737-756

Gaffney DJ, Keightley PD (2006) Genomic selective constraints in murid noncoding DNA. PLoS Genet 2:e204

Haddrill PR, Charlesworth B, Halligan DL, Andolfatto P (2005) Patterns of intron sequence evolution in Drosophila are dependent upon length and GC content. Genome Biol 6:R67

Haddrill PR, Halligan DL, Tomaras D, Charlseworth B (2007) Reduced efficacy of selection in regions of the Drosophila genome that lack crossing over. Genome Biol 8:R18

Halligan DL, Keightley PD (2006) Ubiquitous selective constraints in the Drosophila genome revealed by a genome-wide interspecies comparison. Genome Res 16:875-884

Hardison RC, Roskin KM, Yang S, et al. (2003) Covariation in frequencies of substitution, deletion, transposition, and recombination during eutherian evolution. Genome Res 13:13-26

Hellmann I, Ebersberger I, Ptak SE, Pääbo S, Przeworski M (2003) A neutral explanation for the correlation of diversity with recombination rates in humans. Am J Hum Genet 72:1527-1535

Hey J, Kliman RM (2002) Interactions between natural selection, recombination and gene density in the genes of Drosophila. Genetics 160:595-608

Hill WG, Robertson A (1966) The effect of linkage on limits to artificial selection. Genet Res 8:269-294

Jakubczak JL, Xiong Y, Eickbush TH (1990) Type I (R1) and type II (R2) ribosomal DNA insertions of Drosophila melanogaster are retrotransposable elements closely related to those of Bombyx mori. J Mol Biol 212:37-52

Jensen MA, Charlesworth B, Kreitman M (2002) Patterns of genetic variation at a chromosome 4 locus of Drosophila melanogaster and D. simulans. Genetics 160:493-507

Jordan IK, Rogozin IB, Glazko GV, Koonin EV (2003) Origin of a substantial fraction of human regulatory sequences from transposable elements. Trends Genet 19:68-72

Kapitonov VV, Jurka J (1999) DNAREP1_DM. Repbase update release 3.4. Available at: http://www.girinst.org/Repbase_ Updata.html

Kapitonov VV, Jurka J (2003) Molecular paleontology of transposable elements in the Drosophila melanogaster genome. Proc Natl Acad Sci USA 100:6569-6574

Kaplan NL, Hudson RR, Langley CH (1989) The "hitch-hiking effect" revisited. Genetics 123(4):887-899

Kazazian HH (2004) Mobile elements: drivers of genome evolution. Science 303(5664):1626-1632
Kimura M (1980) A simple method for estimating evolutionary rates of base substitutions through comparative studies of nucleotide sequences. J Mol Evol 16:111-120

Kirby DA, Muse SV, Stephan W (1995) Maintenance of pre-mRNA secondary structure by epistatic selection. Proc Natl Acad Sci USA 92:9047-9051

Kliman RM, Hey J (1993) Reduced natural selection associated with low recombination in Drosophila melanogaster. Mol Biol Evol 10:1239-1258

LePage DF, Church DM, Millie E, Hassold TJ, Conlon RA (2000) Rapid generation of nested chromosomal deletions on mouse chromosome 2. Proc Natl Acad Sci USA 97:10471-10476

Lercher MJ, Hurst LD (2002) Human SNP variability and mutation rate are higher in regions of high recombination. Trends Genet 18:337-340

Marais G, Domazet-Loso T, Tautz D, Charlesworth B (2004) Correlated evolution of synonymous and nonsynonymous sites in Drosophila. J Mol Evol 59:771-779

Maynard-Smith J, Haigh J (1974) The hitch-hiking effect of a favorable gene. Genet Res 23:23-35

McDonald JF (1993) Evolution and consequences of transposable elements. Curr Opin Genet Dev 3:855-864

McVean GA, Vieira J (2001) Inferring parameters of mutation, selection and demography from patterns of synonymous site evolution in Drosophila. Genetics 157:245-257

Moriyama EN, Powell JR (1996) Intraspecific nuclear DNA variation in Drosophila. Mol Biol Evol 13:261-277

Moriyama EN, Powell JR (1997) Synonymous substitution rates in Drosophila: mitochondrial versus nuclear genes. J Mol Evol 45:378-391

Mozer BA, Benzer S (1994) Ingrowth by photoreceptor axons induces transcription of a retrotransposon in the developing Drosophila brain. Development 120:1049-1058

Pardue ML, DeBaryshe PG (2003) Retrotransposons provide an evolutionarily robust non-telomerase mechanism to maintain telomeres. Annu Rev Genet 37:485-511

Petrov DA, Hartl DL (1999) Patterns of nucleotide substitution in Drosophila and mammalian genomes. Proc Natl Acad Sci USA 96:1475-1479

Presgraves DC (2005) Recombination enhances protein adaptation in Drosophila melanogaster. Curr Biol 15:1651-1656

Pyatkov KI, Shostak NG, Zelentsova ES, et al. (2002) Penelope retroelements from Drosophila virilis are active after transformation of Drosophila melanogaster. Proc Natl Acad Sci USA 99:16150-16155

Quesneville H, Bergman CM, Andrieu O, et al. (2005) Combined evidence annotation of transposable elements in genome sequences. PLoS Comput Biol 1:166-175

Shapiro JA, Huang W, Zhang C, Hubisz MJ, Lu J, Turissini DA, Fang S, Wang HY, Hudson RR, Nielsen R, Chen Z, Wu CI (2007) Adaptive genic evolution in the Drosophila genomes, Proc Natl Acad Sci USA 104(7):2271-2276

Singh ND, Petrov DA (2004) Rapid sequence turnover at an intergenic locus in Drosophila. Mol Biol Evol 21:670-680

Singh ND, Arndt PF, Petrov DA (2005a) Genomic heterogeneity of background substitutional patterns in Drosophila melanogaster. Genetics 169:709-722

Singh ND, Davis JC, Petrov DA (2005b) Codon bias and noncoding GC content correlate negatively with recombination rate on the Drosophila X chromosome. J Mol Evol 61:315-324

Slawson EE, Shaffer CD, Malone CD, et al. (2006) Comparison of dot chromosome sequences from $D$. melanogaster and $D$. virilis reveals an enrichment of DNA transposon sequences in heterochromatic domains. Genome Biol 7:R15

Thompson JD, Higgins DG, Gibson TJ (1994) CLUSTAL W: improving the sensitivity of progressive multiple sequence 
alignment through sequence weighting, positions-specific gap penalties and weight matrix choice. Nucleic Acids Res 22:46734680

van de Lagemaat LN, Landry JR, Mager DL, Medstrand P (2003) Transposable elements in mammals promote regulatory variation and diversification of genes with specialized functions. Trends Genet 19:530-536

Venter JC, Adams MD, Myers EW, et al. (2001) The sequence of the human genome. Science 291:1304-1351

Wang J, Keightley PD, Johnson T (2006) MCALIGN2: faster, accurate global pairwise alignment of non-coding DNA sequences based on explicit models of indel evolution. BMC Bioinform 7:292

Waterston RH, Lindblad-Toh K, Birney E, et al. (2002) Initial sequencing and comparative analysis of the mouse genome. Nature 420:520-562
White SE, Habera LF, Wessler SR (1994) Retrotransposons in the flanking regions of normal plant genes: a role for copia-like elements in the evolution of gene structure and expression. Proc Natl Acad Sci USA 91:11792-11796

Yang H-P, Hung T-L, You T-L, Yang T-H (2006) Genomewide comparative analysis of the highly abundant transposable element DINE-1 suggests a recent transpositional burst in Drosophila yakuba. Genetics 173(1):189-196

Yi S, Summers TJ, Pearson NM, Li W-H (2004) Recombination has little effect on the rate of sequence divergence in pseudoautosomal boundary 1 among humans and great apes. Genome Res 14:37-43 